\title{
DISK-CYCLICITY AND CODISK-CYCLICITY OF CERTAIN SHIFT OPERATORS
}

\author{
Yu-XiA LiAng AND ZE-HuA ZHOU
}

\begin{abstract}
In this paper we characterize the disk-cyclicity and codisk-cyclicity of the bilateral weighted shifts on Hilbert space $\ell^{2}(\mathbb{Z}, \mathscr{K})$ with weight sequence $\left\{A_{n}\right\}_{n \in \mathbb{Z}}$ of positive invertible diagonal operators on a separable complex Hilbert space $\mathscr{K}$, respectively. At last, we establish similar results for the disk-cyclic and codisk-cyclic shift operator $B$ on $L^{2}(\beta)$ defined by $B f_{j}=$ $f_{j-1}, j \in \mathbb{Z}$, where $\left\{f_{j}\right\}_{j \in \mathbb{Z}}$ is a basis of $L^{2}(\beta)$.
\end{abstract}

Mathematics subject classification (2010): 47A16, 47B37.

Keywords and phrases: Disk-cyclic, codisk-cyclic, weighted shifts.

\section{REFERENCES}

[1] A. S. ATEF, On G-cyclicity of operators, Thesis, The Islamic University of Gaza, 2007.

[2] F. BAyART, É. MAtheron, Hypercyclic operators failing the Hypercyclicity Criterion on classical Banach spaces, J. Funct. Anal. 250 (2007) 426-441.

[3] F. Bayart, É. Matheron, Dynamics of Linear Operators, Camberidge University Press, 2009.

[4] R. M. Gethner, J. H. Shapiro, Universal vectors for operators on spaces of holomorphic functions, Proc. Amer. Math. Soc. 100 (1987) 281-288.

[5] K. G. Grosse-Erdmann, Hypercyclic and chaotic weighted shifts, Studia. Math. 139 (2000) 47-68.

[6] K. G. Grosse-Erdmann, A. P. Manguillot, Linear Chaos, Springer, New York, 2011.

[7] M. HazariKa, S. C. ARora, Hypercyclic operator weighted shifts, Bull. Korean Math. Soc. 41 (2004) 589-598.

[8] Z. Z. JAmIL, Cyclic Phenomena of operators on Hilbert space, Ph. D. Thesis, University of Baghdad, 2002.

[9] Z. Z. Jamil, M. Helal, Equivalent between the Criterion and the Three Open Set's Conditions in Disk-Cyclicity, Int. J. Contemp. Math. Sciences. 8 (2013) 257-261.

[10] Z. Z. JAMIL, A. G. NAOUM, Disk-cyclic and weighted shifts operators, International J. of Math. Sci. and Engg. Appls. (IJMSEA), 7 (2013) 375-388.

[11] C. KiTAI, Invariant Closed Sets for Linear Operators, Ph. D. Thesis, University of Toronto, 1982.

[12] Y. X. LiAnG AND Z. H. ZHOU, Hereditarily hypercyclicity and supercyclicity of weighted shifts, J. Korean Math. Soc. 51 (2) (2014) 363-382.

[13] Y. X. LiANG AND Z. H. ZHOU, Disjoint supercyclic powers of weighted shifts on weighted sequence spaces, Turkish J. Math. 38 (2014) 1007-1022.

[14] Y. X. LiANG AND Z. H. ZHOU, Hypercyclic behaviour of multiples of composition operators on the weighted Banach space, Bull. Belg. Math. Soc. Simon Stevin, 21 (3) (2014) 385-401.

[15] Y. X. LiAnG AND Z. H. ZHOU, Supercyclic tuples of the adjoint weighted composition operators on Hilbert spaces, Bull. Iranian Math. Soc. 41 (1) (2015) 121-139.

[16] H. N. SALAS, Supercyclicity and weighted shifts, Studia Math. 135 (1999) 55-74.

[17] B. Yousefi And A. Farrokhinia, On the hereditarily hypercyclic operators, J. Korean Math. Soc. 43 (2006) 1219-1229.

[18] L. ZHANG, Z. H. ZHOU, Disjoint mixing weighted backward shifts on the space of all complex valued square summable sequences, J. Comput. Anal. Appl. 16 (4) (2014) 618-625. 